\title{
Effect of Integrated Weed Management Practices against Weed Control in Cluster Bean during Summer
}

\author{
V. G. Magar*, A. M Musmade, B. T Patil and M. N. Bhalekar \\ Department of Horticulture, MPKV, Rahuri (M.S), India \\ *Corresponding author
}

\section{A B S T R A C T}

Keywords

Clusterbean, Imazethapyr, Imazamox, Total weed count, Weed control efficiency

\section{Article Info}

Accepted:

15 November 2019

Available Online:

10 December 2019

\begin{abstract}
A field experiment on the "Effect of Integrated Weed Management practices against weed control in cluster bean" was undertaken during summer 2015 and 2016.The various weeds observed in cluster bean crop during summer season were, viz., Cyperus rotundus, Cynodon dactylon, Commelina benghalensis, Parthenium hysterophorus, Portula caoleracea, Amarathus viridis, Trianthema portulacastrum, Euphorbia hrita, Diger amuricata, Panmicumis achne. Phylanthus niruri, Tridax procumbens and Dinebra retroflexa. Based on two year experimentation, it was found that, during summer of 2015, 2016 and pooled treatment weed free check reported significantly highest weed control efficiency than rest of all the treatments as there was total eradication of weeds which resulted in achieving 100\% weed control efficiency. Post emergence application of herbicides in integration with the hand weeding gave better control of weeds during crop growth period which lowered the total weed population and ultimately resulted in achieving higher weed control efficiency. This could be explained based on the fact that, maximum uptake and better assimilation of herbicides was pronounced as soon as weeds emerged. Less weed intensity and its dry weight in integrated weed control treatments compared to weedy check may also one of the reasons of higher WCE with these treatments. Pooled data of two years indicated that significantly maximum pod yield was recorded due to increase values of yield attributing characters due to favorable environment in the root zone resulting absorption of more water and nutrient as less cropweed competition during critical stage.
\end{abstract}

\section{Introduction}

"A year's seeding is seven year's weeding" and thus Indian agriculture has been defined as a "confrontation with weeds". The welfare of mankind is highly dependent on farmer's ability to control the growth of weeds. Thus, it is necessary to concentrate more on weeding out the undesirables than for any other activity related to increasing agricultural production. Weeds pose most serious problem in legume crops because of the liberal use of farm yard manure, chemical fertilizers and frequent irrigations that help the weeds to grow vigorously. It has been well established that losses from weeds accounts for 45 per cent 
more than when compared to insect pests and diseases of about 30 and 20 per cent, respectively (Rao, 1983). Jain and Singh (2000) stated that an unchecked weed growth in clusterbean caused 47 per cent reduction in seed yield. In order to obtain higher seed yield of clusterbean the crop should be kept free from weeds for the first 30 days after sowing. Weed control is an essential part of all crop production systems. Critical period of cropweed competition in clusterbean is about 20 to 30 days after sowing, during this period, weeds reduce yield by competing with crops for water, nutrients, and sunlight. Season long competition with weeds in clusterbean causes severe yield reduction ranging from $29-48$ per cent and severity may even be higher (70-98 $\%)$ depending on the weed infestation. Hand weeding is a traditional and effective method of weed control, but untimely rains, unavailability of labour at peak time and increasing labour cost are the main limitations of manual weeding. Under such situations, the only alternative that needs to be explored is the use of suitable herbicides which may be effective and economically viable (Gupta 1984). All this add to high cost of production. Under such situations, use of suitable herbicides alone or integrated with hand weeding needs to be explored as an effective and economical method of weed management. So proper weed control method, is the prime need and very much essential to give the herbicide usage its due share to obtain maximum productivity.

\section{Materials and Methods}

The experiment was conducted during summer of 2015, 2016 at the All India Coordinated Research Project on Vegetable Crops, Department of Horticulture, Mahatma Phule Krishi Vidyapeeth, Rahuri (Maharashtra). There were ten treatments laid out in Randomized Block Design with three replications. The allocation of treatments in the replication was done by random method. Clusterbean variety Phule Guar (RHRCB-10) was used for experiment. The experiment consisted of ten integrated weed management treatments viz. $\mathrm{T}_{1}$ : Imazethapyr $10 \% \mathrm{SL} \mathrm{PoE}$ $75 \mathrm{~g} \mathrm{ha}^{-1}$ at $15 \mathrm{DAS}, \mathrm{T}_{2}$ : Imazethapyr $10 \% \mathrm{SL}$ PoE $100 \mathrm{~g} \mathrm{ha}^{-1}$ at $15 \mathrm{DAS}, \mathrm{T}_{3}$ : Imazethapyr 10 $\%$ SL PoE $75 \mathrm{~g} \mathrm{ha}^{-1}$ at $15 \mathrm{DAS}+$ Hand weeding at $30 \mathrm{DAS}, \mathrm{T}_{4}$ :Imazethapyr $10 \% \mathrm{SL}$ PoE $100 \mathrm{~g} \mathrm{ha}^{-1}$ at 15 DAS + Hand weeding at 30 DAS, T 5 : Imazamox $35 \%$ + Imazethapyr $35 \%, 70 \%$ WG PoE $60 \mathrm{~g} \mathrm{ha}^{-1}$ at $15 \mathrm{DAS} \mathrm{T}_{6}$ : Imazamox $35 \%$ + Imazethapyr $35 \%, 70 \%$ WG PoE $70 \mathrm{~g} \mathrm{ha}^{-1}$ at $15 \mathrm{DAS}, \mathrm{T}_{7}$ : Imazamox $35 \%$ + Imazethapyr $35 \%, 70 \%$ WG PoE $60 \mathrm{~g}$ $\mathrm{ha}^{-1}$ at $15 \mathrm{DAS}+$ Hand weeding at $30 \mathrm{DAS}$, $\mathrm{T}_{8}$ :Imazamox $35 \%$ + Imazethapyr $35 \%, 70 \%$ WG PoE $70 \mathrm{~g} \mathrm{ha}^{-1}$ at 15 DAS + Hand weeding at 30 DAS, $\mathrm{T}_{9}$ :Weed free check, and $\mathrm{T}_{10}$ :Weedy check (Control). Important weed species associated with clusterbean crop in the experimental plot were recorded and identified. The treatment wise total weed count was recorded and expressed as number $\mathrm{m}^{2}$ and the total weed count data were subjected to square root transformation $\sqrt{ }(\mathrm{X}+1)$ before statistical analysis. Thereafter treatment wise total dry matter of weed was recorded and expressed as grams at 75 DAS.

The weed dry matter data was subjected to square root transformation $\sqrt{ }(\mathrm{X}+1)$ before statistical analysis. According to Mani et al., (1973) weed control efficiency as a derived parameter out of weed population/density per unit area for studying treatment performance in weed control. Weed Control Efficiency can be worked out taking in to consideration the reduction in weed population in treated plot over weed population in unweeded check. Weed control efficiency at 30, 45, 60 and 75 DAS was worked out based on weed count $\mathrm{m}^{2}$ by adopting the formula,

$W C E=\frac{W P_{C}-W P_{T}}{W P_{C}} \times 100 \%$ 
Where, $\mathrm{WP}_{\mathrm{C}}-$ Weed population $\mathrm{m}^{2}$ in control (unwedded) plot

$\mathrm{WP}_{\mathrm{T}}-$ Weed population $\mathrm{m}^{2}$ in treated plot

The yield obtained per net plot was converted into yield per hectare on an area basis and expressed in $\mathrm{q}$ ha ${ }^{1}$.

\section{Results and Discussion}

The weed flora associated with clusterbean crop was found during summer, viz., Cyperus rotundus, Cynodon dactylon, Commelina benghalensis, Parthenium hysterophorus, Portula caoleracea, Amarathus viridis, Trianthema portulacastrum, Euphorbia hrita, Diger amuricata, Panmicumisachne. Phylanthus niruri, Tridax procumbens and Dinebra retroflexa.

Total weed count number per $\mathrm{m}^{2}$ at 30,45 , 60 and 75 DAS

All the weed control treatments significantly reduced total weed count number per $\mathrm{m}^{2}$ as compared to weedy check at 30, 45, 60 and 75 DAS during summer of 2015, 2016 and pooled, the data is presented in Table 1. There were no weeds in weed free treatment since the weeds were being removed and this treatment was kept weed free throughout the growing period, hence it recorded zero weeds and weedy check (control) noticed highest total weed count per $\mathrm{m}^{2}$ at 30, 45, 60 and 75 DAS during summer of 2015, 2016 and pooled. At $30 \mathrm{DAS}$, there was reduction in of total weed count number per $\mathrm{m}^{2}$ in almost all treatments apart from weedy check (control). The significantly lowest weed count number per $\mathrm{m}^{2}(9.67,8.00$ and 8.83 , respectively) was recorded with treatment $\mathrm{T}_{6}$ (Imazamox $35 \%+$ Imazethapyr 35,70 \% WG PoE $70 \mathrm{~g} \mathrm{ha}^{-1}$ at 15 DAS) than other treatments during summer 2015, 2016 and pooled, respectively. It was at par with treatments $\mathrm{T}_{8}$ (Imazamox $35 \%+$
Imazethapyr $35 \%, 70 \%$ WG PoE $70 \mathrm{~g} \mathrm{ha}^{-1}$ at 15 DAS + Hand weeding at 30 DAS) (12.33, 10.33 and 11.33, respectively) and $T_{5}$ (Imazamox $35 \%$ + Imazethapyr $35 \%, 70 \%$ WG PoE $60 \mathrm{~g} \mathrm{ha}^{-1}$ at $\left.15 \mathrm{DAS}\right)(13.00,10.00$ and 11.50 , respectively).These results are in great analogous with the results reported by Patilet al., (2014), Sangwan (2014), Hassan et al., (2015), Prasanna et al., (2015), Singh et al., (2016) and Shyamlal et al., (2017).

\section{Weed dry matter (g) at 75 DAS}

The weed dry matter $\mathrm{g} \mathrm{m}^{-2}$ was significantly influenced by the various weed control treatments. The data regarding weed dry matter $\mathrm{m}^{-2}$ at 75 DAS is presented in Table 2. During summer of 2015, 2016 and pooled results were noted significantly lower weed dry matter i.e. zero with treatment $\mathrm{T}_{9}$ (weed free check). This could be attributed to control of weeds by hand weeding at regular intervals which resulted in reduced dry matter production of weeds.

The data on weed dry matter during summer of 2015, 2016 and pooled results shows that, the treatments of herbicides used alone and used in integration with hand weeding, treatment $\mathrm{T}_{8}$ (Imazamox $35 \%$ + Imazethapyr $35 \%, 70 \%$ WG PoE $70 \mathrm{~g} \mathrm{ha}^{-1}$ at $15 \mathrm{DAS}+$ Hand weeding at 30 DAS) recorded significantly lowest weed dry matter (4.20, 4.87 and $4.53 \mathrm{~g}$, respectively) than other treatments. However, it was at par with treatment $\mathrm{T}_{7}$ (Imazamox $35 \%$ + Imazethapyr $35 \%, 70 \%$ WG PoE $60 \mathrm{~g} \mathrm{ha}^{-1}$ at $15 \mathrm{DAS}+$ Hand weeding at 30 DAS) (4.57, 5.93 and $5.25 \mathrm{~g}$, respectively) and which followed by $\mathrm{T}_{4}$ (Imazethapyr $10 \%$, SL PoE $100 \mathrm{~g} \mathrm{ha}^{-1}$ at 15 DAS + Hand weeding at 30 DAS $)(6.87$, 8.30 and $7.58 \mathrm{~g}$, respectively) and $\mathrm{T}_{3}$ (Imazethapyr $10 \%$, SL PoE $75 \mathrm{~g} \mathrm{ha}^{-1}$ at 15 DAS + Hand weeding at 30 DAS $)(8.30,9.30$ and $8.80 \mathrm{~g}$, respectively). All weed control treatments gave lower dry matter when 
compared with untreated control i.e. weedy check $\left(\mathrm{T}_{10}\right)$ at 75 DAS. This might be attributed to less density of weeds due to application of herbicide alone and in integration with hand weeding which resulted in lowering the emergence and interrupted growth of weeds this leads to lower weed intensity in weed control treatments. These results indicate the effect of weed control treatment on various morphological traits of weeds and finally reduce the total dry matter production.

This has also been demonstrated by Sumanth Kumar (2005), Dhaker et al., (2009), Singh et al., (2013), Patil et al., (2014), Sangwan (2014), Gupta et al., (2015), Hassan et al., (2015), Singh et al., (2016).

Weed control efficiency $(\%)$ at $30,45,60$, 75 DAS

The weed control efficiency represents efficiency of weeds controlled by different weed management treatments in comparison with weedy check. The weed control efficiency was significantly influenced due to different weed management treatments at different stages. The data with respect to weed control efficiency (\%) worked out at 30, 45, 60 and 75 DAS, during summer of 2015, 2016 and pooled results are presented in Table 3 .

The $100 \%$ weed control efficiency was recorded in treatment $\mathrm{T}_{9}$ (weed free check) at $30,45,60$ and 75 DAS during summer of 2015, 2016 and pooled data. This is mainly due to total elimination of weeds at the critical stages of crop growth. Significantly reduced weed density resulting in higher weed control efficiency. However, the lowest weed control efficiency was observed under $\mathrm{T}_{10}$ (weedy check) in both the seasons of 2015 and 2016, this might be due to the high weed density.
These results found conformity with Kamble et al., (2017).

Among the weed control treatments, treatment $\mathrm{T}_{6}$ (Imazamox $35 \%$ + Imazethapyr $35 \%, 70$ $\%$ WG PoE $70 \mathrm{~g} \mathrm{ha}^{-1}$ at 15 DAS) recorded significantly highest weed control efficiency (91.63, 93.97 and $92.80 \%$, respectively) than the rest of treatments at 30 DAS during summer of 2015, 2016 and pooled respectively. It was found at par with treatment $\mathrm{T}_{8}$ (Imazamox $35 \%$ + Imazethapyr $35 \%, 70 \%$ WG PoE $70 \mathrm{~g} \mathrm{ha}^{-1}$ at $15 \mathrm{DAS}+$ Hand weeding at 30 DAS) and $\mathrm{T}_{5}$ (Imazamox $35 \%$ + Imazethapyr $35 \%, 70 \%$ WG PoE 60 $\mathrm{g} \mathrm{ha}^{-1}$ at 15 DAS) respectively during summer of 2015, 2016 and pooled. At 45 DAS, significantly highest weed control efficiency (98.99, 98.83 and $98.91 \%)$ was noticed under treatment $\mathrm{T}_{8}$ (Imazamox $35 \%$ + Imazethapyr $35 \%, 70 \%$ WG PoE $70 \mathrm{~g} \mathrm{ha}^{-1}$ at 15 DAS + Hand weeding at 30 DAS) during summer of 2015, 2016 and pooled data, respectively. However it was at par with application of treatments $\mathrm{T}_{7}$ (Imazamox $35 \%$ + Imazethapyr $35 \%, 70 \%$ WG PoE $60 \mathrm{~g} \mathrm{ha}^{-1}$ at $15 \mathrm{DAS}+$ Hand weeding at 30 DAS) $(98.71,98.60$ and $98.65 \%$, respectively), $\mathrm{T}_{4}$ (Imazethapyr $10 \%$, SL PoE $100 \mathrm{~g} \mathrm{ha}^{-1}$ at 15 DAS + Hand weeding at 30 DAS) (98.46, 98.60 and $98.53 \%$, respectively) and $\mathrm{T}_{3}$ (Imazethapyr $10 \%$, SL PoE $75 \mathrm{~g} \mathrm{ha}^{-1}$ at $15 \mathrm{DAS}+$ Hand weeding at 30 DAS) (97.94, 97.89 and $97.92 \%$, respectively). The data pertaining to weed control efficiency at 60 and 75 DAS presented in Table 3 revealed that similar trend was observed as that of 45 DAS during summer of 2015, 2016 and pooled.

These results are in agreement with the findings of Singh et al., (2001), Dungarwalet al., (2002), Sumanth Kumar (2005), Sheteet al., (2007), Vaghasia and Nadiyadhara (2013), Patilet al., (2014). 
Table.1 Effect of different weed management treatments on total weed count number $\mathrm{m}^{-2}$ at $30,45,60$ and 75 DAS in cluster bean during summer

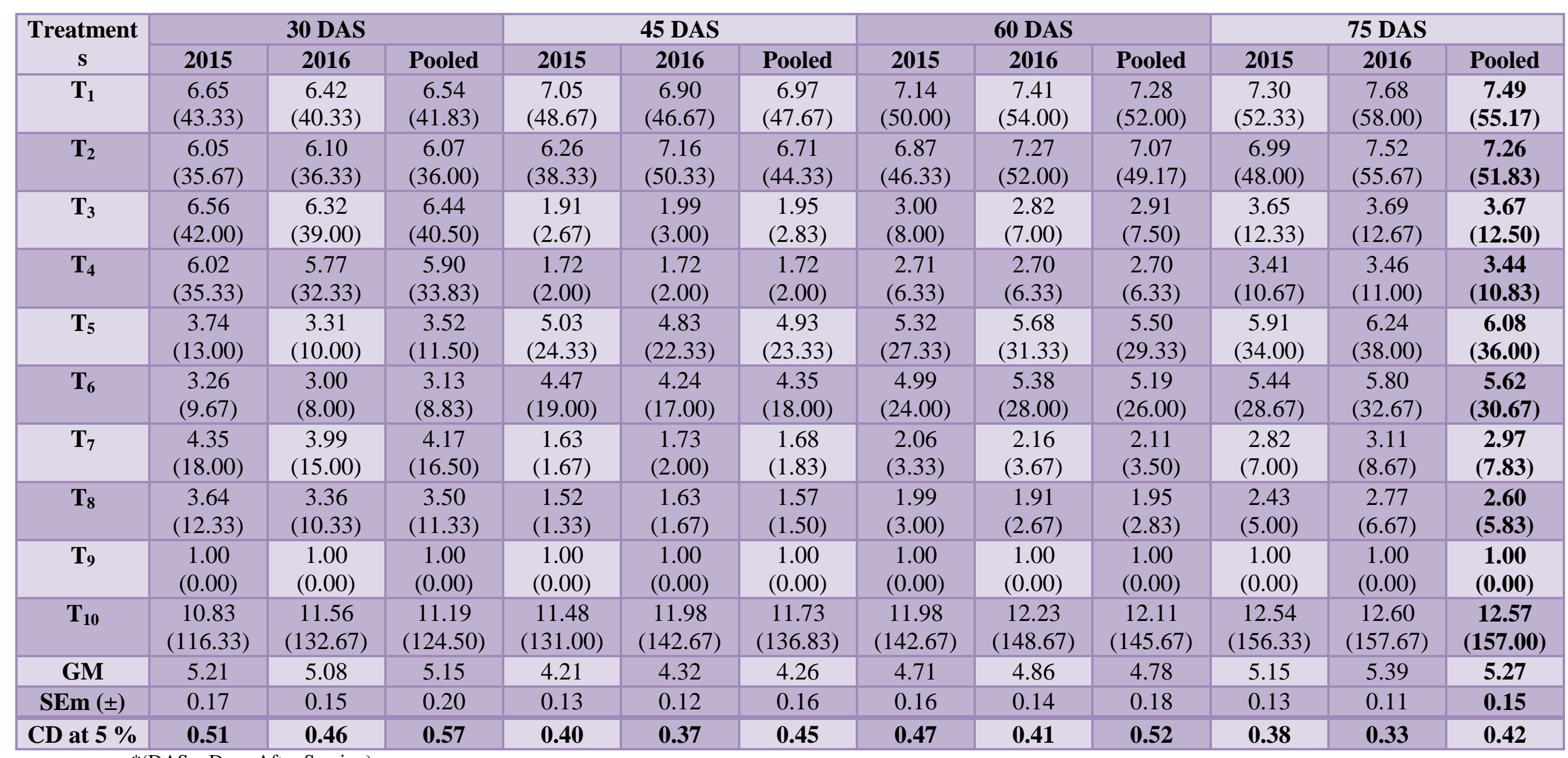

*(DAS - Days After Sowing)

*Figures in parenthesis are original values while figures outside the parenthesis are square root transformed $\sqrt{\boldsymbol{x}+1}$ values.

Treatments : $\mathbf{T}_{\mathbf{1}^{-}}$Imazethapyr $10 \%$ SL PoE $75 \mathrm{~g} \mathrm{ha}^{-1}$ at $15 \mathrm{DAS}, \mathbf{T}_{\mathbf{2}^{-}}$Imazethapyr $10 \%$ SL PoE $100 \mathrm{~g} \mathrm{ha}^{-1}$ at $15 \mathrm{DAS}^{-} \mathbf{T}_{\mathbf{3}^{-}} \mathrm{T}_{1}+\mathrm{Hand}$ weeding at $30 \mathrm{DAS}$, $\mathbf{T}_{\mathbf{4}^{-}}$ $\mathrm{T}_{2}+$ Hand weeding at $30 \mathrm{DAS}, \mathbf{T}_{5^{-}}$Imazamox $35 \%+$ Imazethapyr $35 \%, 70 \%$ WG PoE $60 \mathrm{~g} \mathrm{ha}^{-1}$ at 15 DAS, $\mathbf{T}_{6^{-}}$Imazamox $35 \%+$ Imazethapyr $35 \%, 70 \%$ WG PoE $70 \mathrm{~g} \mathrm{ha}^{-1}$ at $15 \mathrm{DAS}, \mathbf{T}_{\mathbf{7}}-\mathrm{T}_{5}+$ Hand weeding at $30 \mathrm{DAS}, \mathbf{T}_{\mathbf{8}^{-}} \mathrm{T}_{6}+$ Hand weeding at $30 \mathrm{DAS}, \mathbf{T}_{\mathbf{9}}$ - Weed free check, $\mathbf{T}_{\mathbf{1 0}}{ }^{-}$Weedy check $(\mathrm{Control})$. 
Table.2 Effect of different weed management treatments on weed dry matter $\mathrm{g} \mathrm{m}^{-2}$ at 75 DAS in cluster bean during summer

\begin{tabular}{|c|c|c|c|}
\hline \multirow[t]{2}{*}{ Treatments } & \multicolumn{3}{|c|}{ Summer } \\
\hline & 2015 & 2016 & Pooled \\
\hline $\mathbf{T}_{1}$ & $\begin{array}{c}5.82 \\
(33.00)\end{array}$ & $\begin{array}{c}5.47 \\
(29.00)\end{array}$ & $\begin{array}{c}5.65 \\
(31.00)\end{array}$ \\
\hline $\mathbf{T}_{2}$ & $\begin{array}{c}5.37 \\
(28.00)\end{array}$ & $\begin{array}{c}5.54 \\
(29.67)\end{array}$ & $\begin{array}{c}5.45 \\
(28.83)\end{array}$ \\
\hline $\mathbf{T}_{\mathbf{3}}$ & $\begin{array}{c}3.04 \\
(8.30)\end{array}$ & $\begin{array}{c}3.21 \\
(9.30)\end{array}$ & $\begin{array}{c}3.13 \\
(8.80) \\
\end{array}$ \\
\hline $\mathbf{T}_{4}$ & $\begin{array}{c}2.79 \\
(6.87)\end{array}$ & $\begin{array}{c}3.05 \\
(8.30)\end{array}$ & $\begin{array}{c}2.92 \\
(7.58)\end{array}$ \\
\hline $\mathbf{T}_{5}$ & $\begin{array}{c}4.83 \\
(22.33)\end{array}$ & $\begin{array}{c}4.61 \\
(20.23)\end{array}$ & $\begin{array}{c}4.72 \\
(21.28) \\
\end{array}$ \\
\hline $\mathbf{T}_{6}$ & $\begin{array}{c}4.62 \\
(20.37)\end{array}$ & $\begin{array}{c}4.83 \\
(22.33)\end{array}$ & $\begin{array}{c}4.73 \\
(21.35)\end{array}$ \\
\hline $\mathbf{T}_{7}$ & $\begin{array}{c}2.36 \\
(4.57)\end{array}$ & $\begin{array}{c}2.63 \\
(5.93)\end{array}$ & $\begin{array}{c}2.49 \\
(5.25)\end{array}$ \\
\hline $\mathbf{T}_{8}$ & $\begin{array}{c}2.28 \\
(4.20)\end{array}$ & $\begin{array}{c}2.42 \\
(4.87)\end{array}$ & $\begin{array}{c}2.35 \\
(4.53)\end{array}$ \\
\hline $\mathbf{T}_{9}$ & $\begin{array}{c}1.00 \\
(0.00)\end{array}$ & $\begin{array}{c}1.00 \\
(0.00)\end{array}$ & $\begin{array}{c}1.00 \\
(0.00)\end{array}$ \\
\hline $\mathbf{T}_{10}$ & $\begin{array}{c}9.76 \\
(94.27)\end{array}$ & $\begin{array}{c}9.91 \\
(97.27)\end{array}$ & $\begin{array}{c}9.84 \\
(95.77)\end{array}$ \\
\hline GM & 4.19 & 4.27 & 4.23 \\
\hline SEm ( \pm$)$ & 0.12 & 0.10 & 0.13 \\
\hline CD at $5 \%$ & 0.35 & 0.29 & 0.38 \\
\hline
\end{tabular}

*Figures in parenthesis are original values while figures outside the parenthesis are square root transformed $\sqrt{x+1}$ values.

Treatments : $\mathbf{T}_{\mathbf{1}^{-}}$Imazethapyr $10 \%$ SL PoE $75 \mathrm{~g} \mathrm{ha}^{-1}$ at 15 DAS , $\mathbf{T}_{2^{-}}$Imazethapyr $10 \%$ SL PoE $100 \mathrm{~g} \mathrm{ha}^{-1}$ at 15

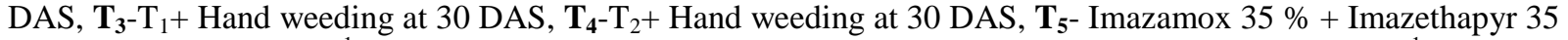
$\%, 70 \%$ WG PoE $60 \mathrm{~g} \mathrm{ha}^{-1}$ at 15 DAS, T$^{-}$Imazamox $35 \%$ + Imazethapyr $35 \%, 70 \%$ WG PoE $70 \mathrm{~g} \mathrm{ha}^{-1}$ at 15 DAS,

$\mathbf{T}_{7}-\mathrm{T}_{5}+$ Hand weeding at 30 DAS, $\mathbf{T}_{\mathbf{8}}-\mathrm{T}_{6}+$ Hand weeding at 30 DAS, $\mathbf{T}_{\mathbf{9}^{-}}$Weed free check, $\mathbf{T}_{\mathbf{1 0}}$ - Weedy check 
Table.3 Effect of different weed management treatments on weed control efficiency (\%) at 30, 45, 60 and 75 DAS in cluster bean for summer

\begin{tabular}{|c|c|c|c|c|c|c|c|c|c|c|c|c|}
\hline \multirow{2}{*}{$\begin{array}{c}\text { Treatmen } \\
\text { ts }\end{array}$} & \multicolumn{3}{|c|}{30 DAS } & \multicolumn{3}{|c|}{45 DAS } & \multicolumn{3}{|c|}{60 DAS } & \multicolumn{3}{|c|}{ 75DAS } \\
\hline & 2015 & 2016 & Pooled & 2015 & 2016 & Pooled & 2015 & 2016 & Pooled & 2015 & 2016 & Pooled \\
\hline$T_{1}$ & 62.64 & 69.58 & 66.11 & 62.66 & 67.23 & 64.94 & 64.92 & 63.62 & 64.27 & 66.52 & 63.19 & 64.86 \\
\hline $\mathbf{T}_{2}$ & 69.32 & 72.68 & 71.00 & 70.76 & 64.67 & 67.72 & 67.58 & 65.04 & 66.31 & 69.36 & 64.68 & 67.02 \\
\hline $\mathbf{T}_{3}$ & 63.76 & 70.52 & 67.14 & 97.94 & 97.89 & 97.92 & 94.38 & 95.28 & 94.83 & 94.86 & 93.65 & 94.25 \\
\hline $\mathbf{T}_{4}$ & 69.43 & 75.53 & 72.48 & 98.46 & 98.60 & 98.53 & 95.55 & 95.74 & 95.64 & 96.39 & 93.84 & 95.12 \\
\hline $\mathbf{T}_{5}$ & 88.86 & 92.49 & 90.67 & 81.36 & 84.33 & 82.85 & 80.76 & 78.89 & 79.83 & 78.16 & 75.92 & 77.04 \\
\hline $\mathbf{T}_{6}$ & 91.63 & 93.97 & 92.80 & 85.40 & 88.05 & 86.72 & 83.11 & 81.14 & 82.12 & 81.67 & 79.26 & 80.46 \\
\hline $\mathbf{T}_{7}$ & 84.59 & 88.75 & 86.67 & 98.71 & 98.60 & 98.65 & 97.68 & 97.54 & 97.61 & 96.80 & 95.55 & 96.18 \\
\hline $\mathbf{T}_{8}$ & 89.37 & 92.22 & 90.80 & 98.99 & 98.83 & 98.91 & 97.91 & 98.21 & 98.06 & 97.45 & 96.38 & 96.92 \\
\hline $\mathbf{T}_{9}$ & 100.00 & 100.00 & 100.00 & 100.00 & 100.00 & 100.00 & 100.00 & 100.00 & 100.00 & 100.00 & 100.00 & 100.00 \\
\hline $\mathbf{T}_{10}$ & 0.00 & 0.00 & 0.00 & 0.00 & 0.00 & 0.00 & 0.00 & 0.00 & 0.00 & 0.00 & 0.00 & 0.00 \\
\hline GM & 71.96 & 75.57 & 73.77 & 79.42 & 79.82 & 79.62 & 78.19 & 77.55 & 77.87 & 78.12 & 76.25 & 77.19 \\
\hline $\operatorname{SEm}( \pm)$ & 1.63 & 1.28 & 1.79 & 1.01 & 0.89 & 1.17 & 1.08 & 1.10 & 1.33 & 0.93 & 0.83 & 1.08 \\
\hline CD at $5 \%$ & 4.83 & 3.80 & 5.14 & 2.99 & 2.66 & 3.35 & 3.21 & 3.26 & 3.82 & 2.76 & 2.46 & 3.09 \\
\hline
\end{tabular}

*(DAS - Days After Sowing)


$\mathrm{T}_{2}+$ Hand weeding at 30 DAS, $\mathbf{T}_{5^{-}}$Imazamox $35 \%$ + Imazethapyr $35 \%, 70 \%$ WG PoE $60 \mathrm{~g}^{-1}$ at 15 DAS, $\mathbf{T}_{6^{-}}$Imazamox $35 \%+$ Imazethapyr $35 \%, 70 \%$ WG PoE $70 \mathrm{~g} \mathrm{ha}^{-1}$ at 15 DAS, $\mathbf{T}_{\mathbf{7}^{-}} \mathrm{T}_{5}+$ Hand weeding at 30 DAS, $\mathbf{T}_{\mathbf{8}^{-}} \mathbf{T}_{6}+$ Hand weeding at 30 DAS, $\mathbf{T}_{\mathbf{9}^{-}}$Weed free check, $\mathbf{T}_{\mathbf{1 0}}$ - Weedy check $($ Control). 
Table.4 Effect of different weed management treatments on yield hectare ${ }^{-1}(\mathrm{q})$ in cluster bean during summer

\begin{tabular}{|c|c|c|c|}
\hline \multirow{2}{*}{ Treatments } & \multicolumn{3}{|c|}{ Yield hectare $^{-1}(\mathbf{q})$} \\
\cline { 2 - 4 } & $\mathbf{2 0 1 5}$ & $\mathbf{2 0 1 6}$ & Pooled \\
\hline $\mathbf{T}_{\mathbf{1}}$ & 48.05 & 52.69 & $\mathbf{5 0 . 3 7}$ \\
\hline $\mathbf{T}_{\mathbf{2}}$ & 52.09 & 56.86 & $\mathbf{5 4 . 4 8}$ \\
\hline $\mathbf{T}_{\mathbf{3}}$ & 97.50 & 81.05 & $\mathbf{8 9 . 2 7}$ \\
\hline $\mathbf{T}_{\mathbf{4}}$ & 102.07 & 82.44 & $\mathbf{9 2 . 2 5}$ \\
\hline $\mathbf{T}_{\mathbf{5}}$ & 68.77 & 67.14 & $\mathbf{6 7 . 9 5}$ \\
\hline $\mathbf{T}_{\mathbf{6}}$ & 78.19 & 68.28 & $\mathbf{7 3 . 2 4}$ \\
\hline $\mathbf{T}_{\mathbf{7}}$ & 105.65 & 97.57 & $\mathbf{1 0 1 . 6 1}$ \\
\hline $\mathbf{T}_{\mathbf{8}}$ & 109.01 & 112.03 & $\mathbf{1 1 0 . 5 2}$ \\
\hline $\mathbf{T}_{\mathbf{9}}$ & 117.26 & 117.97 & $\mathbf{1 1 7 . 6 1}$ \\
\hline $\mathbf{T}_{\mathbf{1 0}}$ & 24.68 & 33.30 & $\mathbf{2 8 . 9 9}$ \\
\hline $\mathbf{G M}$ & 80.33 & 76.93 & $\mathbf{7 8 . 6 3}$ \\
\hline SEm ( $)$ & 2.29 & 2.91 & $\mathbf{3 . 2 1}$ \\
\hline CD at 5 \% & $\mathbf{6 . 8 0}$ & $\mathbf{8 . 6 5}$ & $\mathbf{9 . 2 0}$ \\
\hline
\end{tabular}

*(DAS - Days After Sowing)

Treatments : $\mathbf{T}_{\mathbf{1}^{-}}$Imazethapyr $10 \% \mathrm{SL}$ PoE $75 \mathrm{~g} \mathrm{ha}^{-1}$ at $15 \mathrm{DAS}, \mathbf{T}_{\mathbf{2}^{-}}$Imazethapyr $10 \% \mathrm{SL} \mathrm{PoE} 100 \mathrm{~g} \mathrm{ha}^{-1}$ at 15 DAS, $\mathbf{T}_{3}-\mathrm{T}_{1}+$ Hand weeding at $30 \mathrm{DAS}, \mathbf{T}_{\mathbf{4}}-\mathrm{T}_{2}+$ Hand weeding at $30 \mathrm{DAS}, \mathbf{T}_{5^{-}}$Imazamox $35 \%+$ Imazethapyr 35 $\%, 70 \%$ WG PoE $60 \mathrm{~g} \mathrm{ha}^{-1}$ at 15 DAS, $\mathbf{T}_{6^{-}}$Imazamox $35 \%$ + Imazethapyr $35 \%, 70 \%$ WG PoE $70 \mathrm{~g} \mathrm{ha}^{-1}$ at 15 DAS, $\mathbf{T}_{\mathbf{7}}-\mathrm{T}_{5}+$ Hand weeding at 30 DAS, $\mathbf{T}_{\mathbf{8}}-\mathrm{T}_{6}+$ Hand weeding at 30 DAS, $\mathbf{T}_{\mathbf{9}}$ - Weed free check, $\mathbf{T}_{\mathbf{1 0}}$ - Weedy check (Control).

\section{Pod yield ha ${ }^{-1}(q)$}

The data on pod yield ha ${ }^{-1}$ (q) of cluster bean during summer as influenced by various treatments of weed control are furnished in Table 4.

Perusal to data, an average yield of 80.33 and $76.93 \mathrm{q} \mathrm{ha}^{-1}$ was noticed during 2015 and 2016, respectively. Pooled results reported the average yield of $78.63 \mathrm{q} \mathrm{ha}^{-1}$. Pod yield of cluster bean during both the years and pooled over the years varied significantly due to weed control measures. Significantly superior pod yield was recorded in $\mathrm{T}_{8}$ (Imazamox $35 \%+$ Imazethapyr $35 \%, 70 \%$ WG PoE $70 \mathrm{~g} \mathrm{ha}^{-1}$ at 15 DAS + Hand weeding at 30 DAS $)(109.01 \mathrm{q}$ $\mathrm{ha}^{-1}$ ) which was at par with $\mathrm{T}_{7}$ (Imazamox 35 $\%$ + Imazethapyr $35 \%, 70 \%$ WG PoE $60 \mathrm{~g}$ $\mathrm{ha}^{-1}$ at 15 DAS + Hand weeding at 30 DAS) $\left(105.65 \mathrm{q} \mathrm{ha}^{-1}\right)$ and significantly superior over rest of the integrated weed control treatments except $\mathrm{T}_{9}$ (weed free check) during summer 2015.Significantly higher pod yield was recorded in treatment $\mathrm{T}_{8}$ (Imazamox $35 \%+$ Imazethapyr $35 \%, 70 \%$ WG PoE $70 \mathrm{~g} \mathrm{ha}^{-1}$ at 15 DAS + Hand weeding at 30 DAS) (112.03 $\mathrm{q} \mathrm{ha}^{-1}$ ) which was superior over rest of the integrated weed control measures during summer 2016.Pooled data of two years indicated that significantly maximum pod yield was recorded at $\mathrm{T}_{8}$ (Imazamox $35 \%+$ Imazethapyr $35 \%, 70 \%$ WG PoE $70 \mathrm{~g} \mathrm{ha}^{-1}$ at 15 DAS + Hand weeding at 30 DAS) (110.52 $\mathrm{q} \mathrm{ha}{ }^{-1}$ ) as compared with other integrated weed management practices. This might be due to increase values of yield attributing characters due to favorable environment in the root zone resulting absorption of more water and nutrient as less crop-weed competition during critical stage. These findings also corroborate the results reported by Sumanth 
Kumar (2005), Singh et al., (2008), Lhungdim et al., (2013a), Singh et al., (2013), Vaghasia and Nadiyadhara (2013), Patil et al., (2014), Sangwan (2014) and Patil et al., (2017).

From the present study it can be concluded that, application of Imazamox $35 \%+$ Imazethapy $35 \%, 70 \% \mathrm{WG}$ PoE $70 \mathrm{~g} \mathrm{ha}^{-1}$ at 15 DAS with hand weeding at $30 \mathrm{DAS}\left(\mathrm{T}_{8}\right)$ is most effective treatment to minimize the weed density, lowering the weed dry matter than rest of the treatments except weed free check during summer. Application of Imazamox 35 $\%$ + Imazethapyr $35 \%$, $70 \%$ WG PoE $70 \mathrm{~g}$ $\mathrm{ha}^{-1}$ at $15 \mathrm{DAS}+$ Hand weeding at $30 \mathrm{DAS}$ $\left(\mathrm{T}_{8}\right)$ recorded higher weed control efficiency during summer season. Weed free check recorded significantly highest pod yield per hectare in cluster bean followed by application of Imazamox $35 \%+$ Imazethapyr35 \%,70 \% WG PoE $70 \mathrm{~g} \mathrm{ha}^{-1}$ at $15 \mathrm{DAS}+$ Hand weeding at $30 \mathrm{DAS}\left(\mathrm{T}_{8}\right)$ during summer.

\section{References}

Dhaker, Hemraj, Mundra, S.L. and Jain, N.K. 2009. Weed Management in Clusterbean [Cyamopsis tetragonoloba (L.) Taub.]. Indian J. Weed Sci., 41 (3 and 4): 224-227.

Dungarwal, H.S., Chaplot, P.C. and Nagda, B.L. 2002. Chemical weed control in clusterbean (Cyamopsis tetragonoloba L.). Indian J. Weed Sci., 34(3\&4): 208212.

Gupta, O.P. 1984. Critical Period of Weed Crop Competition Scientific Weed Management in the Tropics and Subtropics, Today and Tomorrow's Printers and Publishers, New Delhi.

Gupta, Versha, Singh, S.P. and. Yadav, R.S. 2015. Yield performance and nutrient uptake as influenced by integrated weed management in cluster bean. Indian J. Weed Sci., 47(1): 82-84.

Hassan, D., Barla, S., Thakur, H.R., Puran,
A.N. and Upasani, R.R. 2015.Studies on time application of imazethapyr and its ready mix combination with imazamox against weeds in blackgram. $25^{\text {th }}$ Asian-Pacific Weed Science Society Conference on "Weed Science for Sustainable Agriculture, Environment and Biodiversity", Hyderabad, INDIA during 13-16 October. (2015): pp. 238.

Jain, A.K. and Singh, S.K. 2000. Integrated weed management in cluster bean. Haryana J. Hort. Sci., 2: 146-149.

Kamble, A.B., Danawale, N.J. and Rajendrakumar. 2017. Integrated weed management in $\mathrm{Bt}$ cotton. Indian $J$. Weed Sci., 49(4): 405-408.

Lhungdim, J., Singh, Y. and Singh, R.P. 2013a. Integration of Chemical and Manual Weed Management on Weed Density, Yield and Production Economics of Lentil (Lens culinaris Medikus). International J. Bioresource and Stress Management, 4(4): 593-598.

Mani, V.S., Pandita, M.L., Gautam, K.C. and Bhagwandas. 1973. Weed killing chemicals in potato cultivation. Indian Farming, 23: 7-13.

Patil, A.G., Halepyati, A.S. and Chittapur, B.M. 2017. Efficacy of Sequential application of Herbicides on soybean growth and yield in north eastern transitional zone of Karnataka. International Archive of Applied Sci. and Technol., 8 (1): 32-37

Patil, B.T., Bhalekar, M.N. and Shinde, K.G. 2014. Weed management in cluster bean (Cyamopsis tetragonoloba (L.) Taub). J. Agric. Res. and Technol., 39(3): 501-504.

Prasanna, B., Goverdhan, M., Sridevi, S. and Venkataramana, M. 2015.Bioefficacy of herbicides and integrated weed management practices in groundnut. 25th Asian Pacific Weed Science 
Society Conference on "Weed Science for Sustainable Agriculture, Environment and Biodiversity", Hyderabad, INDIA during 13-16 October, 2015. pp. 212.

Rao, V.S. 1983. Principles of weed science, Oxford and IBH Publishing Co. New Delhi. pp. 24-42.

Sangwan, Meenakshi. 2014. Efficacy of imazethapyr + imazamox (Ready mix) in cluster bean and its residual effect on mustard in two texturally different soils. M.Sc. (Agriculture) Thesis submitted to College of Agriculture CCS Haryana Agriculture University Hisar (India).

Shete, B.T., Patil, H.M. and Kolekar, P.T. 2007. Effect of cultural practices and post emergence herbicides against weed control in soybean. International J. Agric. Sci., 3(2): 273-275.

Shyamlal, Kewat, M.L. and Suryavanshi, T. 2017. Weed indices as influenced by propaquizafop and imazethapyr mixture in soybean. International $J$. Curr. Microbiol. App. Sci., 6(8): 31093115.

Singh, A., Ahlawat, I.P.S. and Saraf, C.S.
2001. Studies on weed control in clusterbean (Cyamopsis tetragonoloba (L.). Indian J. Agron., 31(3): 269-272.

Singh, G. and Sekhon, H.S. 2013. Integrated weed management in pigeonpea [Cajanus cajan (L.) Millsp.] World J. Agricul. Sci., 9 (1): 86-91.

Singh, S.K., Jain, A.K. and Punia, B.L. 2008. Integrated weed management in clusterbean (Cyamopsis tetragonoloba (L.). Indian J. Agrilcult. Sci., 70(2): $850-852$.

Singh, S.P., Yadav, R.S. and Sharma, V. 2016. Weed control in cluster bean through post-emergence herbicides. Indian $J$. Weed Sci., 48(2): 202-205.

Sumanth Kumar. 2005. Physiological studies on weed control efficiency in cluster bean (Cyamopsis tetragonoloba (L.) Taub). M.Sc. (Agriculture) Thesis submitted to University of Agricultural Sciences, Dharwad (India).

Vaghasia, P.M. and Nadiyadhara, V. 2013.Effect of post- emergence herbicides in groundnut and its residual effect on succeeding crops. International J. Forestry and Crop Improvement, 4(2): 54-58.

\section{How to cite this article:}

Magar, V. G., A. M Musmade, B. T Patil and Bhalekar, M. N. 2019. Effect of Integrated Weed Management Practices against Weed Control in Cluster Bean during Summer. Int.J.Curr.Microbiol.App.Sci. 8(12): 1939-1948. doi: https://doi.org/10.20546/ijcmas.2019.812.232 